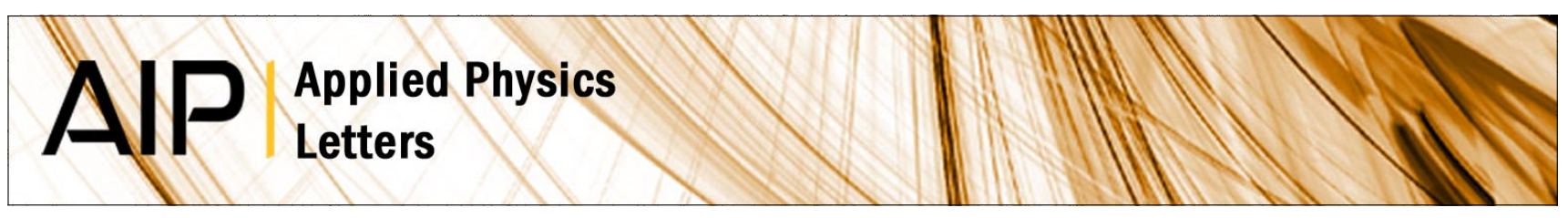

\title{
Optical band-gap determination of nanostructured WO3 film
}

P. P. González-Borrero, F. Sato, A. N. Medina, M. L. Baesso, A. C. Bento et al.

Citation: Appl. Phys. Lett. 96, 061909 (2010); doi: 10.1063/1.3313945

View online: http://dx.doi.org/10.1063/1.3313945

View Table of Contents: http://apl.aip.org/resource/1/APPLAB/v96/i6

Published by the American Institute of Physics.

Additional information on Appl. Phys. Lett.

Journal Homepage: http://apl.aip.org/

Journal Information: http://apl.aip.org/about/about_the_journal

Top downloads: http://apl.aip.org/features/most_downloaded

Information for Authors: http://apl.aip.org/authors

\section{ADVERTISEMENT}

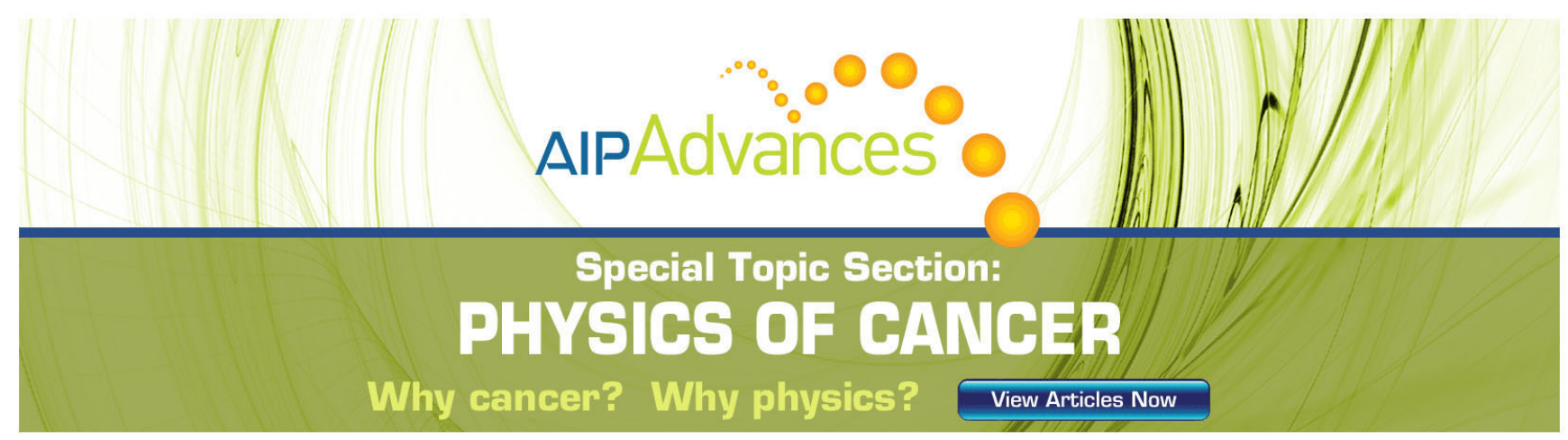




\title{
Optical band-gap determination of nanostructured $\mathrm{WO}_{3}$ film
}

\author{
P. P. González-Borrero, ${ }^{1,2, a)}$ F. Sato, ${ }^{1}$ A. N. Medina, ${ }^{1}$ M. L. Baesso, ${ }^{1}$ A. C. Bento, ${ }^{1}$ \\ G. Baldissera, ${ }^{3}$ C. Persson, ${ }^{3}$ G. A. Niklasson, ${ }^{4}$ C. G. Granqvist ${ }^{4}$ and A. Ferreira da Silva ${ }^{5}$ \\ ${ }^{1}$ Departamento de Física, Universidade Estadual de Maringá, 87020-900 Maringá, Parana, Brazil \\ ${ }^{2}$ Departamento de Física, Universidade Estadual do Centro-Oeste, 85040-080 Guarapuava, Parana, Brazil \\ ${ }^{3}$ Department of Materials Science and Engineering, Royal Institute of Technology, SE-100 44 \\ Stockholm, Sweden \\ ${ }^{4}$ Department of Engineering Sciences, The Angström Laboratory, Uppsala University, P.O. Box 534, \\ S-751 21 Uppsala, Sweden \\ ${ }^{5}$ Instituto de Física, Universidade Federal da Bahia, Campus Universitário de Ondina, 40210-340 Salvador, \\ Bahia, Brazil
}

(Received 18 December 2009; accepted 19 January 2010; published online 9 February 2010)

The optical band-gap energy of a nanostructured tungsten trioxide film is determined using the photoacoustic spectroscopy method under continuous light excitation. The mechanism of the photoacoustic signal generation is discussed. The band-gap energy is also computed by other methods. The absorption coefficient as well as the band-gap energy of three different crystal structures of tungsten trioxide is calculated by a first-principles Green's function approach using the projector augmented wave method. The theoretical study indicates that the cubic crystal structure shows good agreement with the experimental data. (C) 2010 American Institute of Physics. [doi:10.1063/1.3313945]

Tungsten trioxide $\left(\mathrm{WO}_{3}\right)$ films have attracted much interest during the last decade due to their potential applications. Nanostructured $\mathrm{WO}_{3}$ films have been used in eletrochromic (EC) devices such as displays and smart windows. ${ }^{1-3}$ For this reason, a detailed understanding of the optical processes responsible for the EC effect would greatly facilitate the optimization of EC devices. ${ }^{4} \mathrm{WO}_{3}$ is a wideband-gap semiconductor. Its band-gap energy has been mainly measured by optical absorption, varying from about 2.6 to $3.0 \mathrm{eV}^{2,5}$ The band gap of $\mathrm{WO}_{3}$ is certainly of interest for both applied and fundamental aspects. The literature is however somewhat confusing. Values below $3.0 \mathrm{eV}$ have mostly been obtained assuming an indirect band gap.

Taking into account that the understanding of the optical processes responsible for the EC effect is an important parameter in design and optimization of EC devices, and that the band gap energy is one of the most important parameter of semiconductors, we investigate the optical absorption in the region of the fundamental band edge by the photoacoustic spectroscopy (PAS) technique. PAS has been extensively used as a nondestructive method for measuring the optical properties of semiconductors and many other materials. ${ }^{6-10}$ The nonradiative relaxation processes-which are associated with the band structure, defect-related energy loss mechanism, etc. — can be directly and very accurately obtained from the analysis of the PAS spectra. ${ }^{10}$

The optical band-gap energy $\left(E_{g}\right)$ has been determined by the PAS technique using mainly two methods. In the first, the $E_{g}$ value is adopted as the absorption edge obtained from a linear fitting in the plot of the square of the product between the absorption coefficient and the photon energy versus the photon energy for direct band gap, or the plot of the square root of the product between the absorption coefficient and the photon energy versus the photon energy for indirect band gap. ${ }^{11}$ In the second, $E_{g}$ is estimated by the changing of

${ }^{\text {a)} E l e c t r o n i c ~ m a i l: ~ g o n z a l e z @ u n i c e n t r o . b r . ~}$ the derivative near the fundamental absorption edge. ${ }^{7}$

In this letter, we analyze the PA-signal behavior of a nanostructured $\mathrm{WO}_{3}$ film under continuous laser excitation, using an experimental procedure similar to that described in Ref. 12. The influence of the continuous excitation in the mechanisms responsible for the generation of the PA signal is discussed and adopted to determine the band-gap energy directly from the experimental optical absorption spectra. Additionally, the absorption coefficients of three crystal structures of $\mathrm{WO}_{3}$ are studied theoretically by employing a projector augmented wave (PAW) method within the $\mathrm{GW}_{0}$ approximation to calculate the band-gap energies.

The nanostructured tungsten oxide film, with an approximate thickness of $5.0 \mu \mathrm{m}$, was obtained by depositing a carbox- $\mathrm{WO}_{3}$ mixture onto a conducting glass substrate (15 to $20 \Omega$ per square). The dried film was initially heated at $140{ }^{\circ} \mathrm{C}$ for $15 \mathrm{~min}$ and then sintered in air at $500{ }^{\circ} \mathrm{C}$ for 60 min. Further experimental details can be found in Ref. 13.

PA spectra were obtained in the region between 1.5 and $4.5 \mathrm{eV}(275-820 \mathrm{~nm})$. All the PA spectra were normalized by the PA signal of carbon black powder used as a reference sample to eliminate the spectral characteristic of the optical source. The experimental setup consists basically of a 1000 W Xe-arc lamp source, a monochromator, a chopper with light modulation frequency of $21 \mathrm{~Hz}$ and the sample placed in a conventional PA cell. The PA signal was detected by a Bruel and Kjaer microphone and processed by a 5210 PAR

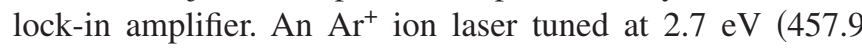
$\mathrm{nm})$ or $3.5 \mathrm{eV}(351 \mathrm{~nm})$ was used as continuous light source. Additional details of experimental conditions are described in Ref. 12. The measurements were carried out in the following way: initially, the PA spectra were taken while the sample was excited only by the modulated light. Then, the spectra were obtained with simultaneous excitation by the continuous laser light. Hereafter, we call this procedure excitation method. 


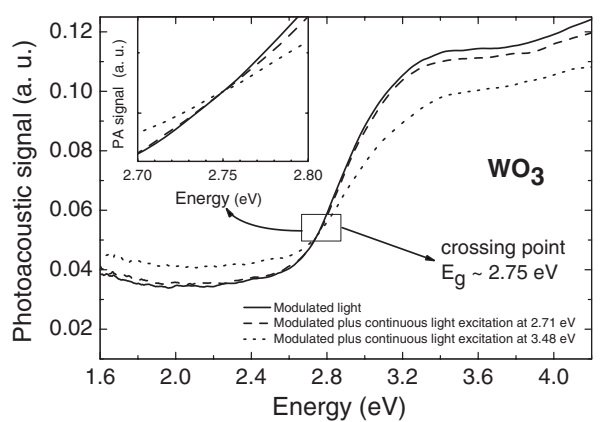

FIG. 1. Room temperature PA signal of the nanostructured $\mathrm{WO}_{3}$ film as a function of photon energy. The continuous line represents the typical PA signal with modulated light. The other curves stand for PA signal under both modulated and continuous excitations with $50 \mathrm{~mW}$ at $2.71 \mathrm{eV}$ (dashed) and $150 \mathrm{~mW}$ at $3.48 \mathrm{eV}$ (dotted). The direct band gap $E_{g}=(2.75 \pm 0.06) \mathrm{eV}$ is obtained from the crossing point of PA signal with and without continuous excitation (shown in the inset)

Figure 1 depicts the spectra for $\mathrm{WO}_{3}$ with or without excitation by continuous laser light as a function of photon energy. The spectrum with modulated light only shows an onset of the optical absorption band around $2.60 \mathrm{eV}$. Using PAS under continuous light excitation we obtained the band gap energy around $2.75 \mathrm{eV}$ in agreement with literature values. The $E_{g}$ is determined by considering the crossing point between modulated and continuous light as described in Ref. 12. Other techniques used to obtain the value of $E_{g}$ present difficulties due to the uncertainties in the determination of the absolute value of optical absorption coefficient in the band-gap edge. By excitation with continuous light, the generated PA signal increases or decreases for energies higher or lower than that of the band gap, respectively. Then, at the band gap position the signal is the same for both measurement procedures. The spectrum with continuous light, shown in Fig. 1, was corrected by making the difference between the measured spectrum and the background obtained scanning the spectrum with the Xe lamp off and the continuous laser light on. Doing in this way, possible contributions from the back scattering can be avoided. To improve accuracy, we have repeated all measurements three times and calculated the respective errors. The final result was obtained by averaging over these three independent results.

As it is known, PAS can provide information about the carrier transport properties if the absorbed energy $\hbar \omega>E_{g}$. In this case, an excess of photoinjected carriers, with energy $\hbar \omega-E_{g}$, is created in the conduction band, followed by electron-phonon collision de-excitation that takes place in the picoseconds scale. Then, as the carriers give off the excess of energy to the lattice, they diffuse through the sample and reestablish equilibrium by recombination with the holes in the valence band. Having this in mind, it is possible to explain the increasing and the decreasing of the generated PA signal with the continuous light excitation around the band gap as the excitation energy varies when compared to the signal with only modulated light. In the case of $\hbar \omega>E_{g}$, the modulated electrons are already in the conduction band when continuous light impinges on the sample. The continuous light, then, may induce these electrons to absorb additional energy resulting in intraband transitions. During this process, there is an increase in the nonradiative relaxation and since the PA signal is inversely proportional to the time of the recombination processes, the generated signal decreases as

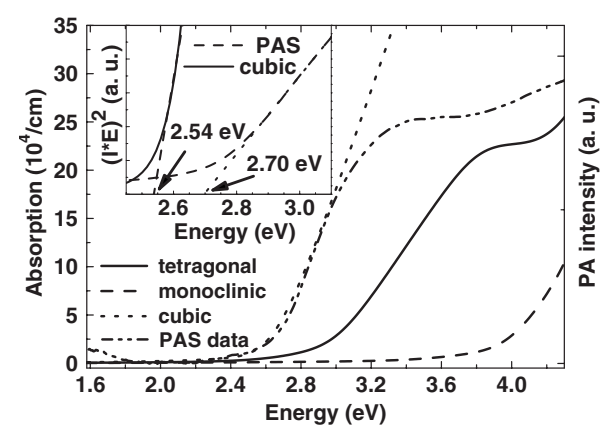

FIG. 2. Total absorption spectra of the $\mathrm{WO}_{3}$ film for monoclinic, tetragonal, and cubic crystalline structures, including a Lorentzian broadening of 0.1 eV. For comparison, the experimental PA curve was also included. The inset shows the relative intensity $(I \bullet E)^{2}$ of $\mathrm{WO}_{3}$ as a function of photon energy near the fundamental absorption edge at room temperature. The dotted line crosses the energy axis at $(2.70 \pm 0.06) \mathrm{eV}$ for PAS data. In the case of the theoretical cubic crystalline structure, $I$ stands for the absorption coefficient, and the dashed line intercepts the abscissa energy at $2.54 \mathrm{eV}$.

shown in Fig. 1. On the other hand, whenever $\hbar \omega<E_{g}$, the modulated light does not have enough energy to excite the electron into the conduction band. When continuous light, however, is switched on additional energy is given to the valence electron allowing them to reach the conduction band. As a consequence of the higher probability of the modulated light to be absorbed by the excess of free carriers, a higher PA signal is generated. This PA-signal behavior, for energies higher or lower than $E_{g}$, is more visible when the continuous light has a higher energy than that of $E_{g}$, as shown in Fig. 1.

The absorption coefficients of $\mathrm{WO}_{3}$ are studied theoretically by employing the PAW method ${ }^{14}$ within the local density approximation (LDA). The LDA is improved by the partially self-consistent $\mathrm{GW}_{0}$ method where the energies are reevaluated in the Green's functions. ${ }^{15}$ For comparison, we also present band structure of LDA $+\mathrm{U}$ with correction of the $\mathrm{W}$ d-states by $\mathrm{U}=4 \mathrm{eV}$ effective on-site Coulomb interaction parameter. Correction of the LDA d-states (i.e., compared with other metal oxides ${ }^{16,17}$ ) is thus taken into account in both $\mathrm{GW}_{0}$ and the LDA+U. We investigate three rather different crystalline structures of $\mathrm{WO}_{3}$, namely, monoclinic $P 2_{1} / c$, tetragonal $P 4 / n m m$ and cubic $P m \overline{3} m{ }^{18}$ The crystal structures are fully relaxed in terms of total energy and forces. Absorption coefficient is obtained with a general energy cut-off of $300 \mathrm{eV}\left(100 \mathrm{eV}\right.$ for the $\mathrm{GW}_{0}$ part) and a $\Gamma$-centered $2 \times 2 \times 2,6 \times 6 \times 6$, and $8 \times 8 \times 8 \mathbf{k}$-mesh, respectively, in the irreducible part of the Brillouin zone.

Figure 2 illustrates the calculated absorption coefficient of the different crystal structures of $\mathrm{WO}_{3}$. For comparison, the experimental PAS curve is also included. As it can be seen, the theoretical cubic crystal structure shows a reasonably good agreement with experimental PAS data when compared to other structures. The beginning absorption value has been estimated as $2.63 \mathrm{eV}$ for the theoretical cubic crystal structure. This value is taken from Fig. 3 considering direct transitions. The onset of the optical absorption band might be slightly underestimated by the theoretical calculations due to temperature effects. The calculation was done at $T=0 \mathrm{~K}$ while the measurements were taken at $300 \mathrm{~K}$. This can be explained by the flat energy dispersion of the energy bands along the (100) direction (Fig. 3). This will certainly increase the measured absorption via phonon-assisted optical transitions at moderate and high temperature. From Fig. 3, it is 


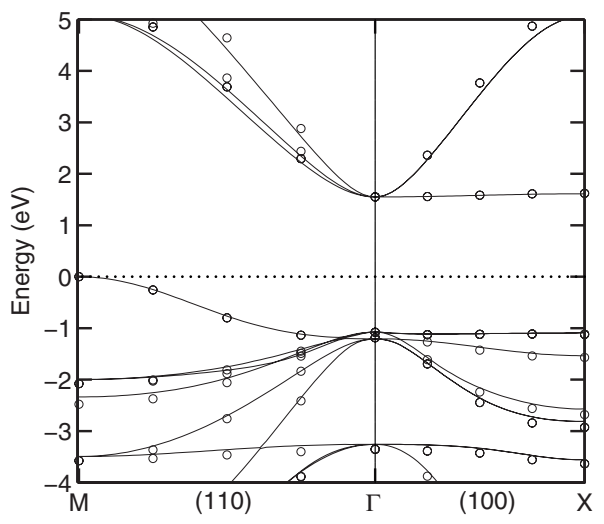

FIG. 3. Electronic bands for $\mathrm{WO}_{3}$ in cubic structure, calculated along the two main symmetry directions. We show both the LDA $+\mathrm{U}$ (solid lines) and the $\mathrm{GW}_{0}$ (marks) results, in which the conduction bands of $\mathrm{LDA}+\mathrm{U}$ have been shifted by $+0.73 \mathrm{eV}$ for better comparison with the $\mathrm{GW}_{0}$ energy bands. Energies are referred to the valence band maximum (dotted line). The band edges along (100) show very flat energy dispersions which will enhance the optical absorption at higher temperature due to phonon-assisted transitions.

clear that the top of the valence band is not at the $\Gamma$-point, and thus the cubic $\mathrm{WO}_{3}$ is an indirect-gap semiconductor.

In order to have a comparison of different methods, we also determined the band-gap energy applying the linear method. It uses the relation for allowed direct transitions $(I \bullet E)^{2}=A\left(E-E_{g}^{L}\right),{ }^{11}$ in which $I$ is the absorption intensity, $A$ is a coefficient and $E$ the photon energy. The value of $E_{g}^{L}$ is obtained by the extrapolation of the best-fit line between $(I \bullet E)^{2}$ and $E$ up to the point where it crosses the axis energy, as shown in Fig. 2 (inset). This method provides the bandgap energy at 2.70 and at $2.54 \mathrm{eV}$ for the experimental PAS curve and the theoretical one, respectively. This theoretical curve refers to the cubic crystal structure. It can be observed that the excitation method shows a higher energy value $(2.75 \mathrm{eV})$.

In summary, we have investigated the optical band-gap energy of a $\mathrm{WO}_{3}$ film by means of PAS using the continuous laser excitation procedure. The results provided an $E_{g}$ value of $(2.75 \pm 0.06 \mathrm{eV})$. The excitation method, besides providing the experimental optical gap, also permitted us to examine the energy transfer processes. The theoretical results for
$\mathrm{WO}_{3}$ with cubic structure showed reasonable agreement with the PAS data as compared to the monoclinic and tetragonal ones.

This work was supported by the Brazilian Agencies FAPESB/PRONEX (Contract No. PNX0005/2007), CAPES, Fundação Araucária, Swedish Research Council (VR), the Swedish Energy Agency (STEM), the European EM ECW Programme EUBRANEX, and the computer centers PDC/ NSC via SNIC/SNAC.

${ }^{1}$ C. G. Granqvist, Handbook of Inorganic Electrochromic Materials (Elsevier, Amsterdam, 1995).

${ }^{2}$ C. G. Granqvist, Sol. Energy Mater. Sol. Cells 60, 201 (2000).

${ }^{3}$ G. A. Niklasson and C. G. Granqvist, J. Mater. Chem. 17, 127 (2007).

${ }^{4}$ L. Berggren and G. A. Niklasson, Appl. Phys. Lett. 88, 081906 (2006).

${ }^{5}$ R. G. Bamwenda, K. Sayama, and H. Arakawa, J. Photochem. Photobiol., A 122, 175 (1999).

${ }^{6}$ T. Ikari, A. Fukuyama, K. Maeda, K. Futagami, S. Shigetomi, and Y. Akashi, Phys. Rev. B 46, 10173 (1992).

${ }^{7}$ A. Ferreira da Silva, N. Veissid, C. Y. An, I. Pepe, N. Barros de Oliveira, and A. V. Batista da Silva, Appl. Phys. Lett. 69, 1930 (1996).

${ }^{8}$ A. K. Ghosh, B. K. Sarkar, and B. K. Chaudhuri, Solid State Commun. 113, 41 (1999).

${ }^{9}$ K. Sakai, T. Kakeno, T. Ikari, S. Shirakata, T. Sakemi, K. Awai, and T. Yamamoto, J. Appl. Phys. 99, 043508 (2006).

${ }^{10}$ Photoacoustic and Thermal Wave Phenomena in Semiconductors, edited by A. Mandelis (North-Holland, New York, 1987).

${ }^{11}$ J. I. Pankove, Optical Processes in Semiconductors (Dover, New York, 1975).

${ }^{12}$ N. G. C. Astrath, F. Sato, F. Pedrochi, A. N. Medina, A. C. Bento, M. L. Baesso, C. Persson, and A. Ferreira da Silva, Appl. Phys. Lett. 89, 231926 (2006).

${ }^{13} \mathrm{H}$. Wang, T. Lindgren, J. He, A. Hagfeldt, and S.-E. Lindquist, J. Phys. Chem. B 104, 5686 (2000).

${ }^{14}$ G. Kresse and D. Joubert, Phys. Rev. B 59, 1758 (1999); P. E. Blöchl, ibid. 50, 17953 (1994).

${ }^{15}$ M. Shishkin and G. Kresse, Phys. Rev. B 75, 235102 (2007); F. Fuchs, J. Furthmüller, F. Bechstedt, M. Shishkin, and G. Kresse, ibid. 76, 115109 (2007)

${ }^{16}$ C. Persson, C. L. Dong, L. Vayssieres, A. Augustsson, T. Schmitt, M. Mattesini, R. Ahuja, J. Nordgren, C. L. Chang, A. Ferreira da Silva, and J.-H. Guo, Microelectron. J. 37, 686 (2006).

${ }^{17}$ A. Ferreira da Silva, I. Pepe, J. P. de Souza, C. Moyses Araujo, C. Persson, R. Ahuja, B. Johansson, C. Y. Yang, and J.-H. Guo, Phys. Scr. T109, 180 (2004); C. Persson and A. Ferreira da Silva, Appl. Phys. Lett. 86, 231912 (2005)

${ }^{18}$ Pearson's Handbook of Crystallographic Data for Intermetallic Phases, 2nd ed., edited by P. Villar and L. Calvert (ASM, Metals Park, OH, 1996). 